\title{
APPLICATION OF ADJOINT SENSITIVITY ANALYSIS TO NUCLEAR REACTOR FUEL ROD PERFORMANCE
}

\author{
Scott J. WILDERMAN and Gary S. WAS \\ University of Michigan, Department of Nuclear Engineering, Ann Arbor, MI 48109, USA
}

Received 28 November 1983

\begin{abstract}
Adjoint sensitivity analysis in nuclear fuel behavior modeling is extended to operate on the entire power history for both Zircaloy and stainless steel cladding via the computer codes FCODE-ALPHA/SS and SCODE/SS. The sensitivities of key variables to input parameters are found to be highly non-intuitive and strongly dependent on the fuel-clad gap status and the history of the fuel during the cycle. The sensitivities of five key variables, clad circumferential stress and strain, fission gas release, fuel centerline temperature and fuel-clad gap, to eleven input parameters are studied. The most important input parameters (yielding significances between 1 and 100) are fabricated clad inner and outer radii and fuel radius. The least important significances (less than 0.01 ) are the time since reactor start-up and fuel burnup densification rate. Intermediate to these are fabricated fuel porosity, linear heat generation rate, the power history scale factor, clad outer temperature, fill gas pressure and coolant pressure. Stainless steel and Zircaloy have similar sensitivities at start-up but these diverge as burnup proceeds due to the effect of the higher creep rate of Zircaloy which causes the system to be more responsive to changes in input parameters. The value of adjoint sensitivity analysis lies in its capability of uncovering dependencies of fuel variables on input parameters that cannot be determined by a sequential thought process.
\end{abstract}

\section{Introduction}

Adjoint sensitivity analysis is a technique for quantitatively determining the dependence of output information on input information in large complex system models. This analysis technique has been applied to several problems in nuclear reactor power operation including the nuclear fuel cycle [1], fuel cycle costs [2] and nuclear fuel behavior modeling [3]. In the latter instance, a complex computer code is constructed using a set of equations that describe the thermo-mechanical behavior of an operating fuel rod subject to a set of input conditions. Execution of the code involves solving these equations in a predetermined sequence to arrive at the specified output information. Sensitivity analysis reveals how, at any time during the operation of the code, a variable changes with respect to a change in any input parameter. Since all variables are, in general, interrelated, all variations must be solved for simultaneously. Further, since they are a function of rod history, they must be updated each time step.

In an earlier paper[3] the use of adjoint sensitivity analysis in the modeling of a Zircaloy-clad fuel rod was described. The fuel behavior code used in the analysis was FCODE-ALPHA, a component of EPRI's
SPEAR-ALPHA code system [4]. The model consisted of a system of 33 equations and 11 parameters. The sensitivity analysis was implemented in the code SCODE [4]. Results were presented for the first time step in the power history.

In this paper, the application of sensitivity analysis to fuel performance is reviewed and extended to include the entire rod power history. The dependence of the sensitivity of any output variable to any input parameter at all points in the power history is thus determined. Both the fuel behavior code and the sensitivity analysis code are extended to accommodate stainless steel fuel cladding (FCODE-ALPHA/SS and SCODE/SS) [5]. Sensitivity analysis is applied to operating histories of stainless steel rods and Zircaloy test rods in the Connecticut Yankee reactor. The importance of various input parameters as they affect key output variables is assessed for both cases. Applications of adjoint sensitivity analysis in reactor operation are discussed.

\section{Background}

In a previous paper [3] the authors developed the formalism for adjoint sensitivity analysis. The following 
is a review of only those aspects needed to understand the extension to the multiple time step problem. The reader is referred ref. [3] for a complete theoretical treatment of adjoint sensitivity analysis in fuel behavior modeling.

All of the equations used to model fuel behavior in FCODE-ALPHA(/SS) can be classified as belonging to either of two categories: Differential equations in which a variable is defined in terms of a relation given for its derivative; and constraint equations in which no derivatives appear and the variable is expressed in terms of other variables and parameters. These can be written as

$\dot{x}_{m}=f_{m}(\boldsymbol{x}, \boldsymbol{y}, \boldsymbol{p}, t), \quad m=1, \ldots, M$,

$y_{n}=g_{n}(x, y, p, t), \quad n=M+1, \ldots, N$,

where

$\boldsymbol{x}=$ variables defined in differential equations,

$\boldsymbol{y}=$ variables defined in constraint equations,

$\boldsymbol{p}=$ set of parameters in the analytic model,

$t=$ time,

$f, g=$ functions describing the dependence of the variables on $x, y, p$ and $t$.

Sensitivities are formed by taking the variations of the differential and constraint equations to yield:

$\delta \dot{x}_{m}=\sum_{m^{\prime}} \frac{\partial f_{m}}{\partial x_{m^{\prime}}} \delta x_{m^{\prime}}+\sum_{n} \frac{\partial f_{m}}{\partial y_{n}} \delta y_{n}+\sum_{k} \frac{\partial f_{m}}{\partial p_{k}} \delta p_{k}$,

$\delta y_{n}=\sum_{m} \frac{\partial g_{n}}{\partial x_{m}} \delta x_{m}+\sum_{n^{\prime}} \frac{\partial g_{n}}{\partial y_{n^{\prime}}} \delta y_{n^{\prime}}+\sum_{k} \frac{\partial g_{n}}{\partial p_{k}} \delta p_{k}$,

where $m=1, \ldots, M ; n=M+1, \ldots, N ;$ and $k=$ $1, \ldots, K$. In our system model, the number of differential and constraint equations describing the thermomechanical behavior of stainless steel clad is different from that for Zircaloy clad. For the Zircaloy case, $M=2$ and $N=33$. The stainless steel model represents the degenerate case of adjoint sensitivity analysis in which $M=0$ and $N=31$. In both instances, however, the number of parameters, $K$, is 11 .

At this point we define the following for the general case:

$(\delta X)_{N}=$ column vector of the $N$ dependent variations:

$\delta x_{1}, \ldots, \delta x_{M}, \delta y_{M+1}, \ldots, \delta y_{N}$.

This vector subsumes both $x$-type and $y$ type variables.

$\left(\delta X^{\prime}\right)_{N}=$ column vector of the $N$ variations with $\delta \dot{x}_{m}$ replacing $\delta x_{m}, m=1, \ldots, M$.

$(\delta P)_{K}=$ column vector of $K$ parameters.

$[A]_{N, N}=$ matrix consisting of the coefficients of the variations in dependent variables (i.e. $\partial f / \partial \boldsymbol{x}, \partial f / \partial \boldsymbol{y}, \partial \boldsymbol{g} / \partial \boldsymbol{x}$ and $\partial \boldsymbol{g} / \partial \boldsymbol{y})$.

$[C]_{N, K}=$ matrix consisting of the coefficients of the variation in parameters of the $N$ equations (i.e. $\partial f / \partial p$ and $\partial g / \partial p$ ). Note that in general, both $[A]_{N, N}$ and $[C]_{N, K}$ will have implicit time dependence through their use of the dynamical variables $x$ and $y$. Then eqs. (2) can be written as

$\left(\delta X^{\prime}\right)_{N}=[A]_{N, N}(\delta X)_{N}+[C]_{N, K}(\delta P)_{K}$.

\section{Sensitivity analysis in the degenerate case}

As mentioned earlier, the model for stainless steel is a special case in which there are no differential equations, $M=0$, so $\left(\delta X^{\prime}\right)_{N}=(\delta X)_{N}$ and eq. (3) reduces to

$(\delta X)_{N}=[A]_{N, N}(\delta X)_{N}+[C]_{N, K}(\delta P)_{K}$.

Collecting terms,

$[1-A]_{N, N}(\delta X)_{N}=[C]_{N, K}(\delta P)_{K}$,

and multiplying eq. (5) by $(\delta P)_{K}^{-1}$

$[1-A]_{N, N}(\delta X)_{N}(\delta P)_{K}^{-1}=[C]_{N, K}(\delta P)_{K}(\delta P)_{K}^{-1}$

Since $(\delta X)_{N}(\delta P)_{K}^{-1}=[\delta X / \delta P]_{N, K}$ and $(\delta P)_{K}(\delta P)_{K}^{-1}$ $=1$, then

$[1-A]_{N, N}[\delta X / \delta P]_{N, K}=[C]_{N, K}$.

Sensitivities can thus be calculated by matrix inversion

$[\delta X / \delta P]_{N, K}=[1-A]_{N, N}^{-1}[C]_{N, K}$.

This is the case for the thermo-mechanical behavior of stainless steel clad fuel. All of the equations defining the output variables are constraint equations and the sensitivities of the $N$ output variables with respect to the $K$ input parameters are determined directly from eq. (8). The stainless steel model consists of 31 equations and 11 parameters.

The description of creep strain is the most likely source of differential type equations. Creep in stainless steel fuel cladding is modeled using a time-hardening rule

$\epsilon_{\mathrm{eq}}=A \sigma_{\mathrm{eq}}[1-\exp (-\phi t / B)]+C \sigma_{\mathrm{eq}} \phi t$,

where $\sigma_{\text {eq }}=$ equivalent stress, $\varphi t=$ fluence and $A, B$, $C=$ constants. Hence, the creep strain is written explicitly as a function of other variables and parameters. Note that creep in stainless steel is due entirely to 
irradiation and there is no direct thermal contribution. Cladding temperature does affect the irradiation creep rate, but not sufficiently (at normal operating temperatures) to act as an independent source of creep.

Thermal creep cannot, however, be ignored in Zircaloy at operating temperatures. The total creep strain is the sum of irradiation and thermal creep components. The thermal creep rate is defined in terms of creep strain, so the creep strain rate will be a function of the total strain. The creep strains are therefore defined by differential variables as [4]

$$
\begin{aligned}
\dot{\epsilon}_{\mathrm{eq}, \text { th }}= & (m / 24)\left(C_{1} C_{2}\right)^{1 / m} \sigma_{\mathrm{eq}}^{n / m} \epsilon_{\mathrm{eq}, \mathrm{th}}^{(m-1) / m} \\
& \times\left[\exp \left(Q_{1} T_{\mathrm{R}}\right)\right]^{1 / m},
\end{aligned}
$$

and

$\dot{\epsilon}_{\mathrm{eq}, \mathrm{irr}}=C_{3} C_{4} \phi^{m_{2}} \sigma_{\mathrm{eq}}^{n_{2}} \exp \left(Q_{2} T_{\mathrm{R}}\right)$

where

$\epsilon_{\text {eq, th }}=$ current value of equivalent thermal creep strain,

$\dot{\epsilon}_{\text {eq,th }}=$ current value of equivalent thermal creep rate,

$\sigma_{\text {eq }}=$ clad equivalent stress,

$\phi \quad=$ fast flux,

$T_{\mathrm{R}}=$ creep corner temperature,

$Q_{1} \quad=$ primary creep thermal coefficient,

$Q_{2}=$ secondary creep thermal coefficient,

$m$ = time exponent in related time hardening rule $(0.3)$,

$n=$ stress exponent in related stress hardening rule (2.76),

$m_{2}=$ secondary creep flux exponent $(0.85)$,

$n_{2}=$ secondary creep stress exponent (1.23),

$C_{1}, C_{2}, C_{3}, C_{4}=$ constants.

In FCODE-ALPHA(/SS) irradiation and thermal creep strains have been renormalized by $\epsilon_{\text {eq,th }}^{1 / m}$ in order to avoid infinite primary creep at reactor start-up.

\section{Adjoint sensitivity analysis for Zircaloy cladding}

We now have two differential equations and so we introduce adjoint functions $\phi_{m}, m=1, \ldots, M$ and $\psi_{n}$, $n=M+1, \ldots, N$, conjugate to the variables $x_{m}$ and $y_{n}$ respectively. We require that these adjoint functions adhere to the adjoint relations

$$
\begin{aligned}
& \dot{\phi}_{m}=-\frac{\partial}{\partial x_{m}}(\boldsymbol{\phi} \cdot \boldsymbol{f}+\psi \cdot \boldsymbol{g}), \quad m=1, \ldots, M, \\
& \psi_{n}=\frac{\partial}{\partial y_{n}}(\boldsymbol{\phi} \cdot \boldsymbol{f}+\psi \cdot \boldsymbol{g}), \quad n=M+1, \ldots, N .
\end{aligned}
$$

Noting that $\phi$ and $\psi$ are functions of $t$ only, we can reduce the adjoint relations to

$$
\begin{aligned}
& -\dot{\phi}_{m}=\boldsymbol{\phi} \cdot \frac{\partial}{\partial x_{m}} f+\psi \cdot \frac{\partial}{\partial x_{m}} \boldsymbol{g}, \quad m=1, \ldots, M \\
& \psi_{n}=\boldsymbol{\phi} \cdot \frac{\partial}{\partial y_{n}} f+\psi \cdot \frac{\partial}{\partial y_{n}} \boldsymbol{g}, \quad n=M+1, \ldots, N .
\end{aligned}
$$

We now define the adjoint row vectors by their transposed vectors

$$
\begin{aligned}
& (\Phi)_{N}^{\mathrm{T}}=\left(\phi_{1}, \phi_{2}, \psi_{M+1}, \ldots, \psi_{N}\right) \\
& \left(\Phi^{\prime}\right)_{N}^{\mathrm{T}}=\left(-\dot{\phi}_{1},-\dot{\phi}_{2}, \psi_{M+1}, \ldots, \psi_{N}\right) .
\end{aligned}
$$

Then the adjoint eq. (13) takes the form

$\left(\Phi^{\prime}\right)_{N}^{\mathrm{T}}=(\Phi)^{\mathrm{T}}[A]_{N, N} \square$

or when transposed

$\left(\Phi^{\prime}\right)_{N}=[A]_{N, N}^{\mathrm{T}}(\Phi)_{N} \square$

where $\left(\Phi^{\prime}\right)_{N}$ and $(\Phi)_{N}$ are now column vectors and $[A]_{N, N}^{\mathrm{T}}$ is the transpose of $[A]_{N, N}$.

In our system two sets of adjoint functions are required and the adjoint equation is expressed as

$\left[\begin{array}{cc}-\dot{\phi}_{1,1} & -\dot{\phi}_{1,2} \\ -\dot{\phi}_{2,1} & -\dot{\phi}_{2,2} \\ \psi_{3,1} & \psi_{3,2} \\ \vdots & \vdots \\ \psi_{33,1} & \psi_{33,2}\end{array}\right]=[A]_{N, N}^{\mathrm{T}}\left[\begin{array}{ll}\phi_{1,1} & \phi_{1,2} \\ \phi_{2,1} & \phi_{2,2} \\ \psi_{3,1} & \psi_{3,2} \\ \vdots & \vdots \\ \psi_{33,1} & \psi_{33,2}\end{array}\right]$,

where now, $\left[\Phi^{\prime}\right]_{N, 2}$ and $[\Phi]_{N, 2}$ are $N \times 2$ matrices since there are two differential equations in our set of equations.

We may, without loss of generality, set the following initial conditions on the adjoint functions

$\left\{\begin{array}{l}\phi_{1,1}(0)=1 \\ \phi_{2,1}(0)=0\end{array}\right\}$ and $\left\{\begin{array}{l}\phi_{1,2}(0)=0 \\ \phi_{2,2}(0)=1\end{array}\right\}$.

This gives for eq. (18)

$\left[\begin{array}{cc}-\dot{\phi}_{1,1} & -\dot{\phi}_{1,2} \\ -\dot{\phi}_{2,1} & -\dot{\phi}_{2,2} \\ \psi_{3,1} & \psi_{3,2} \\ \vdots & \vdots \\ \psi_{33,1} & \psi_{33,2}\end{array}\right]=[A]_{N, N}^{\mathrm{T}}\left[\begin{array}{ll}1 & 0 \\ 0 & 1 \\ \psi_{3,1} & \psi_{3,2} \\ \vdots & \vdots \\ \psi_{33,1} & \psi_{33,2}\end{array}\right]$.

We must now solve eq. (20) for $[\psi]_{3: N, 2}$, to obtain the 
remaining initial conditions for the $\psi$-type adjoint functions from the constraint equations. To do this, we rewrite eq. (20) by decomposing the matrix multiplication into the part multiplying the $2 \times 2$ matrix and the part multiplying the $\psi$ vectors.

$$
[\phi]_{3: N, 2}=[A]_{3: N .1: 2}^{\mathrm{T}}+[A]_{3: N, 3: N}^{\mathrm{T}}[\psi]_{3: N, 2},
$$

where $[\psi]_{3: N, 2}$ is the matrix of the two column vectors $\left.\psi_{3, i}, \psi_{4, i}, \ldots, \psi_{33, i}\right), i=1,2$ and $[A]_{3: N, 1: 2}^{\mathrm{T}}$ are the first and second columns of the $[\mathrm{A}]_{N, N}^{\mathrm{T}}$ matrix including elements from the 3 rd to the $N$ th row. Likewise, $[A]_{3: N, 3: N}^{\mathrm{T}}$ is that part of the $[A]_{N, N}^{\mathrm{T}}$ matrix restricted to elements from the 3 rd to the $N$ th rows and columns. Eq. (21) admits a direct matrix solution for the $\psi$ matrix

$$
[\psi]_{3: N, 2}=\left[1-[A]_{3: N, 3: N}^{\mathrm{T}}\right]^{-1}[A]_{3: N, 1: 2}^{\mathrm{T}} \text {. }
$$

Between eqs. (19) and (22) we have the full set of $2 N$ initial conditions on the $2 N$ adjoint functions of $[\Phi]_{N, 2}$.

\section{Updating the adjoint functions}

At the end of each time step we must update the adjoint functions to track their time evolution. Hence we would like a transformation that does the following

$$
[\Phi(t+\Delta t)]_{N, 2}=[B]_{N, N}[\Phi(t)]_{N, 2},
$$

where the elements of $[\Phi]_{N, 2}$ are known from the previous time step. Attempts to produce this simple type of transformation based on the method described by Christensen et al. [3] have proved unsuccessful. Therefore, a less approximate (and more time consuming) method is required. Two methods have been found which produce comparable results.

\subsection{Iterative updating method}

Using the forward difference approximation we can write

$$
-\phi_{m}(t)=\frac{-\phi_{m}(t+\Delta t)+\phi_{m}(t)}{\Delta t}, \quad m=1, \ldots, M
$$

Substituting into the variational relation (17) for rows $1, \ldots, M$ only, we have

$$
\left[\frac{\Phi(t+\Delta t)+\Phi(t)}{\Delta t}\right]_{1: 2,2}=[A(t)]_{1: 2, N}^{\mathrm{T}}[\Phi(t)]_{N, 2},
$$

which reduces to

$[\Phi(t+\Delta t)]_{1: 2,2}=\left[\Delta t[A(t)]_{1: 2, N}^{\mathrm{T}}-1\right][\Phi(t)]_{N, 2} \cdot$

To a first approximation, we set

$\psi_{n}(t+\Delta t)=\psi_{n}(t), \quad n=M+1, \ldots, N$.

Eq. (26) which determines $[\Phi(t+\Delta t)]_{3: N, 2}$ is combined with the adjoint functions as determined in eq. (25b) to produce a first approximation of $[\Phi(t+\Delta t)]_{N .2}$,

$$
\begin{aligned}
{[\Phi(t+\Delta t)]_{N, 2}=} & {\left[\Delta t[A(t)]_{1: 2, N}^{\mathrm{T}}-1\right][\Phi(t)]_{N, 2} } \\
& +[\Phi(t)]_{3: N, 2}
\end{aligned}
$$

This approximation of $[\Phi(t+\Delta t)]_{N, 2}$ can be combined with the updated matrix $[A(t+\Delta t)]_{N, N}^{\mathrm{T}}$ in the adjoint relation (17) to produce

$\left[\Phi^{\prime}(t+\Delta t)\right]_{N, 2}^{z+1}=[A(t+\Delta t)]_{N, N}^{\mathrm{T}}[\Phi(t+\Delta t)]_{N, 2}^{z}$,

where $z$ is the iteration number. The matrix $[\Phi(t+$ $\Delta t)]_{N, 2}^{2+1}$ is calculated from $\left[\Phi^{\prime}(t+\Delta t)\right]_{N, 2}^{z+1}$ for the next iteration by

$$
\begin{aligned}
& \phi_{m}^{z+1}(t+\Delta t)=\phi_{m}(t)+\left(\dot{\phi}_{m}^{z+1}(t+\Delta t)\right. \\
& \left.\quad+\dot{\phi}_{m}(t)\right) \Delta t / 2, \quad m=1, \ldots, M, \\
& \psi_{n}^{z+1}(t+\Delta t)=\psi_{n}^{z+1}(t+\Delta t), \quad n=M+1, \ldots, N,
\end{aligned}
$$

where $\dot{\phi}_{m}^{z+1}(t+\Delta t)$ and $\psi_{n}^{z+1}(t+\Delta t)$ come from the matrix multiplication in eq. (28). This yields a new set of adjoint functions, $[\Phi(t+\Delta t)]$, for the next iteration. The iteration process is continued until the maximum variation in any adjoint function between iterations falls below $1 \%$.

\subsection{Matrix inversion method}

This technique employs a backward difference scheme along with matrix inversion. We begin with

$\dot{\phi}_{m}(t+\Delta t)=\frac{\phi_{m}(t+\Delta t)-\phi_{m}(t)}{\Delta t}, \quad m=1, \ldots, M$,
$\psi_{n}(t+\Delta t)=\psi_{n}(t+\Delta t), \quad n=M+1, \ldots, N$.

Substituting these relations into the adjoint relation (17) gives

$\left[\begin{array}{c}\phi_{m}(t) / \Delta t \\ -0\end{array}\right]_{N, 2}+\left[\begin{array}{c}-\phi_{m}(t+\Delta t) / \Delta t \\ -\psi_{n}(t+\Delta t)\end{array}\right]_{N, 2}$ 


$$
=[A(t+\Delta t)]_{N, N}^{\mathrm{T}}\left[\begin{array}{l}
\phi_{m}(t+\Delta t) \\
\hdashline \psi_{n}(t+\Delta t)
\end{array}\right]_{N, 2},
$$

where the dashed line is drawn below row $M$ and the notation $\left[\frac{\text { terms }}{0}\right]$ denotes that all matrix elements below row $M$ are zero. Multiplying columns 1 through $M$ of $[A(t+\Delta t)]_{N, N}^{\mathrm{T}}$ by $-\Delta t$ and the rows 1 through $M$ of

$$
\begin{aligned}
& {\left[\begin{array}{c}
\phi_{m}(t+\Delta t) \\
\psi_{n}(t+\Delta t)
\end{array}\right]_{N, 2} \text { by } 1 / \Delta t \text { we have }} \\
& {\left[\begin{array}{c}
-\phi_{m}(t+\Delta t) / \Delta t \\
\hdashline \psi_{n}(t+\Delta t)
\end{array}\right]_{N, 2}+\left[\begin{array}{c}
\phi_{m}(t) / \Delta t \\
0
\end{array}\right]_{N, 2}} \\
& =\left[A^{\prime}(t+\Delta t)\right]_{N, N}^{\mathrm{T}}\left[\begin{array}{c}
-\phi_{m}(t+\Delta t) / \Delta t \\
-\psi_{n}(t+\Delta t)
\end{array}\right]_{N, 2},
\end{aligned}
$$

where $\left[A^{\prime}(t+\Delta t)\right]_{N, N}^{\mathrm{T}}=[A(t+\Delta t)]_{N, N}^{\mathrm{T}}$ with rows 1 $M$ multiplied by $-\Delta t$. Eq. (32) leads directly to a solution by matrix inversion

$$
\begin{aligned}
& {\left[\begin{array}{l}
-\phi_{m}(t+\Delta t) / \Delta t \\
\hdashline \psi_{n}(t+\Delta t)
\end{array}\right]_{N, 2}=\left[\left[A^{\prime}(t+\Delta t)\right]_{N, N}^{\mathrm{T}}-1\right]^{-1}} \\
& {\left[\begin{array}{c}
\phi_{m}(t) / \Delta t \\
\hdashline 0
\end{array}\right]_{N, 2}}
\end{aligned}
$$

Since $\left[A^{\prime}(t+\Delta t)\right]_{N, N}$ and $\phi_{m}(t)$ are known, $\phi_{m}$ and $\psi_{n}$ at $t+\Delta t$ are determined directly.

Both methods described produce comparable solutions for the updated adjoint functions which are then used according to ref. [3] to obtain the sensitivities

$S_{i j}=\frac{\partial X_{i}}{\partial P_{j}}=$ sensitivity of output $X_{i}$ to input $P_{j}$.

A dimensionless form of the sensitivity is the significance, defined by

$$
S I_{i j}=\frac{P_{j}}{X_{i}} \frac{\partial X_{i}}{\partial P_{j}}
$$

and is the percent change in the output variable given a percent change in the input parameter.

Since the output of SCODE(/SS) is a matrix containing over 300 sensitivities, verifying that SCODE(/SS) calculates sensitivities accurately is an enormous task. This is done in the following benchmarking exercise.

\section{Benchmarking sensitivities}

A method has been devised in which a single input parameter is altered slightly from the reference case and an FCODE-SCODE run is made [6]. Such an alteration or "tweak" is performed for each of the eleven input parameters and is followed by an FCODE-SCODE run. The results are used in two ways. First, observed sensitivities from multiple runs of FCODE are calculated and compared with the computed sensitivities produced by SCODE. When these are in agreement, we assume that no problems exist in the modeling or coding of SCODE. However, when they do not agree, further evaluation is required. This is done by assessing the terms comprising the $[A]_{N, N}$ matrix. First, we define the following;

$$
\begin{aligned}
& \Delta P_{k} \quad=\text { small change in the input parameter } P_{k} \text {, } \\
& \Delta V_{i} \quad=\text { change in variable } V_{i} \text { due to a change in } \\
& P_{k} \text {, } \\
& \Delta V_{i} / \Delta P_{k}=\text { observed sensitivity from FCODE run, } \\
& \delta V_{i} / \delta P_{k} \quad=\text { computed sensitivity from SCODE run, } \\
& \partial V_{i} / \partial V_{j}=\text { derivative of variable } V_{i} \text { with respect to } \\
& \text { variable } V_{j} \text { (these terms comprise the } \\
& {[A]_{N, N} \text { matrix), }} \\
& \partial V_{i} / \partial P_{k}=\text { derivative of variable } V_{i} \text { with respect to } \\
& \text { input parameter } \boldsymbol{P}_{k} \text { (these terms comprise } \\
& \text { the }[C]_{N, K} \text { matrix). }
\end{aligned}
$$

We note that we can expand the total derivative of $V_{i}$

Table 1

Problems uncovered in benchmarking SCODE

- INPUT PARAMETER is so large that the observed $\Delta_{\text {OUT }} / \Delta_{\text {IN }}$ is not a valid approximation of the computed $\delta_{\text {OUT }} / \delta_{\text {IN }}$.

- OUTPUT VARIABLE is so small that it is determined more by machine roundoff error than by code calculations.

- FCODE is not solved simultaneously in all convergence loops, but SCODE uses all current values in calculating coefficients, so it is simultaneous.

- The fission gas release model of FCODE is too complex to be model accurately in SCODE. Therefore a simple, easily formulated but less accurate model is used in SCODE.

- The SCODE equations may not be faithful to the FCODE equations. This type error can be corrected by altering SCODE, once the errors are discovered.

- Multiple FCODE runs produces secants, whereas sensitivities analysis produces tangents. (Reducing the increment size improves the agreement.) 
Table 2

Summary of FCODE-SCODE runs

\begin{tabular}{llllll}
\hline $\begin{array}{l}\text { Power } \\
\text { history }\end{array}$ & \multicolumn{2}{l}{ Stainless steel } & & \multicolumn{2}{l}{ Zircaloy } \\
\cline { 2 - 3 } \cline { 5 - 6 } & $\begin{array}{l}\text { Fuel } \\
\text { type A }\end{array}$ & $\begin{array}{l}\text { Fuel } \\
\text { type B }\end{array}$ & & $\begin{array}{l}\text { Fuel } \\
\text { type A }\end{array}$ & $\begin{array}{l}\text { Fuel } \\
\text { type B }\end{array}$ \\
\hline 1 & $\times$ & $\times$ & $\times$ & $\times$ \\
2 & & $\times$ & & $\times$ \\
\hline
\end{tabular}

with respect to $P_{k}$ as follows:

$$
\frac{\mathrm{d} V_{i}}{\mathrm{~d} P_{k}}=\frac{\partial V_{i}}{\partial P_{k}}+\sum_{j=1}^{33} \frac{\partial V_{i}}{\partial V_{j}} \frac{\mathrm{d} V_{j}}{\mathrm{~d} P_{k}}
$$

We now assume that our observed sensitivities are approximations of the derivatives, or

$\frac{\Delta V_{i}}{\Delta P_{k}} \simeq \frac{\mathrm{d} V_{i}}{\mathrm{~d} P_{k}}$

Substituting this into eq. (36) yields

$$
\frac{\Delta V_{i}}{\Delta P_{k}} \simeq \frac{\partial V_{i}}{\partial P_{k}}+\sum_{j=1}^{33} \frac{\partial V_{i}}{\partial V_{j}} \frac{\Delta V_{j}}{\Delta P_{k}} \text {. }
$$

By carrying out the calculation on the right hand side of eq. (37) and comparing it with the observed sensitivity (left hand side of eq. (37)), we can obtain a good estimation of the validity of the terms in the $[A]_{N, N}$ or $[C]_{N, K}$ matrices. Table 1 shows the various categories of problems uncovered by this techniques. It was also found that in general, the open gap case produced readily verifiable sensitivities. However, after a large number of time steps following gap closure, many of the sensitivities begin to oscillate in sign. This behavior was traced to the convergence of the $2 \times 2$ differential adjoint matrix to a singular matrix. A solution to the problem has not yet been found so data is presented for only those portions of the power history in which the sensitivities are well behaved.

\section{Application to stainless steel and Zircaloy clad fuel rod performance}

FCODE-SCODE runs are made for two fuel types, two rod types and on two power histories, table 2 . The power histories are shown in fig. 1 and the rod precharacterization data are given in table 3 . Since the output from sensitivities is so voluminous; 33 output variables $\times 11$ input parameters $\times 300$ time steps $\sim 10^{5}$ numbers per code run, we will focus on the output variables and input parameters given in table 4. First, several examples will be given to illustrate the non-intuitive behavior of sensitivities during the power history. This will be followed by a comparison of sensitivities for Zircaloy and stainless steel cladding and a ranking of input parameter importance as determined by sensitivity analysis.

\subsection{Sensitivity of fuel centerline temperature to fabricated fuel radius, $\delta T_{0} / \delta R_{\mathrm{F}, \mathrm{fab}}$}

The behavior of the fuel centerline temperature in fuel performance is critical since it is largely responsible for all thermally activated processes such as fission gas release, swelling, creep, etc. The fuel fabricated radius is an input parameter that is under the control of the manufacturer and has a strong influence on the fuel temperature throughout the power history. The signifi-

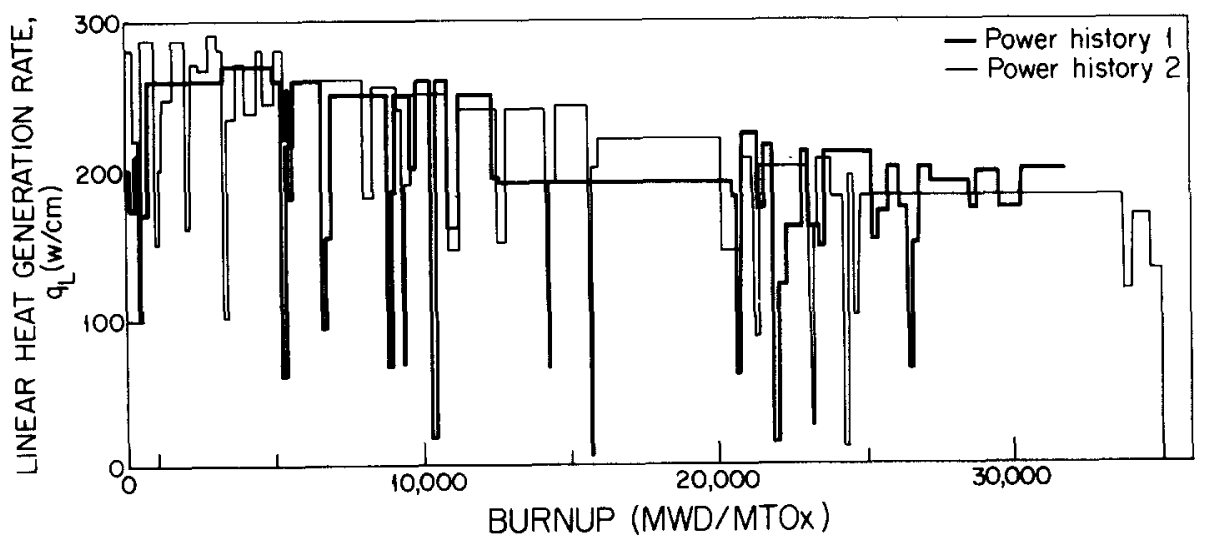

Fig. 1. Power histories applied to Zircaloy and stainless steel clad fuel. 
Table 3

Precharacterization data for fuel types A and B and Zircaloy and stainless steel cladding

\begin{tabular}{|c|c|c|}
\hline & Type A & Type B \\
\hline $\begin{array}{l}\text { bu }_{\text {den }} \text { (burnup densification rate } \\
\text { (MWD/MTOx)) }\end{array}$ & 2000 & 4000 \\
\hline$P_{\text {fab }}$ (fabricated porosity fraction) & 0.0515 & 0.0483 \\
\hline pellet length/diameter & 1.095 & 1.235 \\
\hline \multirow[t]{2}{*}{ Enrichment (\%) } & 4.00 & 3.25 \\
\hline & Zircaloy & stainless steel \\
\hline$R_{\text {Co,fab }}($ clad outer radius $(\mathrm{mm}))$ & $\overline{5.3785}$ & 5.3594 \\
\hline$R_{\mathrm{Cl}, \mathrm{fab}}($ clad inner radius $(\mathrm{mm}))$ & 4.7435 & 4.9403 \\
\hline$R_{\mathrm{f}, \mathrm{fab}}($ fuel radius $(\mathrm{mm}))$ & 4.6292 & 4.8705 \\
\hline$t_{\mathrm{g}}^{\prime}($ radial gap $(\mathrm{mm}))$ & 0.1143 & 0.0698 \\
\hline$\sigma_{y}($ clad yield strength $(\mathrm{MPa}))$ & 276.0 & 482.6 \\
\hline
\end{tabular}

cance of fuel centerline temperature to as-fabricated fuel radius for power history 2 , fuel type $B$, is given in fig. 2. Note that the significance for the stainless steel rod remains negative and decreases over the power history while that for the Zircaloy rod rises to positive values later in life. The sensitivity of fuel centerline temperature, $T_{0}$, to as-fabricated fuel radius, $R_{\mathrm{F}, \mathrm{fab}}$, is strongly coupled to the fuel surface temperature as follows,

$T_{0}=T_{\mathrm{F}}+\int_{0}^{R_{\mathrm{F}}} \frac{q_{\mathrm{L}}(r)}{2 \pi r k_{\mathrm{f}}(r)} \mathrm{d} r$,

where $q_{\mathrm{L}}(r)=$ radially dependent linear heat generation rate, $k_{\mathrm{f}}(r)=$ fuel thermal conductivity, and assuming for simplicity that $q_{\mathrm{L}}$ and $k_{\mathrm{f}}$ are not functions of $r$, then

$T_{0}=T_{\mathrm{F}}+q_{\mathrm{L}} / 4 \pi k_{\mathrm{f}}$.

The fuel surface temperature is given by

$T_{\mathrm{F}}=T_{\mathrm{CI}}+q_{\mathrm{L}} / 2 \pi R_{\mathrm{F}} h_{\mathrm{g}}$,

where

$h_{\mathrm{g}}= \begin{cases}\frac{k_{\mathrm{g}}}{t_{\mathrm{g}}+\lambda_{\mathrm{g}}+\delta_{\mathrm{g}}}, & \text { gap open, } \\ \frac{k_{\mathrm{g}}}{\lambda_{\mathrm{g}}+\delta_{\mathrm{g}}}+a_{\mathrm{g}} p_{\mathrm{c}}, & \text { gap closed, }\end{cases}$

and

$T_{\mathrm{CI}}=$ clad inner temperature,

$q_{\mathrm{L}}=$ linear heat generation rate,

$R_{\mathrm{F}}=$ restrained hot fuel radius,

$h_{\mathrm{g}}=$ gap conductance,

$k_{\mathrm{g}}=$ gap gas conductivity,
Table 4

Output variables and input parameters selected for analysis

\begin{tabular}{|c|c|c|}
\hline \multicolumn{3}{|c|}{ a. Output variables } \\
\hline Variable & Units & Description \\
\hline $\begin{array}{l}\boldsymbol{\epsilon}_{\boldsymbol{\theta}, \mathbf{c}} \\
\boldsymbol{\sigma}_{\boldsymbol{\theta}} \\
G_{\mathrm{Rel}} \\
T_{0} \\
t_{\mathrm{g}}^{\prime}\end{array}$ & $\begin{array}{l}(-) \\
(\mathrm{Pa}) \\
(\mathrm{mol}) \\
\left({ }^{\circ} \mathrm{C}\right) \\
(\mathrm{m})\end{array}$ & $\begin{array}{l}\text { circumferential clad creep strain } \\
\text { circumferential clad stress } \\
\text { fission gas release since start-up } \\
\text { fuel centerline temperature } \\
\text { unrestrained fuel/clad radial gap b }\end{array}$ \\
\hline \multicolumn{3}{|c|}{ b. Input parameters } \\
\hline Paramete & Units & Description \\
\hline $\begin{array}{l}R_{\mathrm{Cl}, \mathrm{fab}} \\
R_{\mathrm{F}, \mathrm{fab}} \\
P_{\mathrm{fab}} \\
\mathrm{bu}_{\mathrm{den}} \\
p_{\mathrm{I}, \mathrm{fab}}\end{array}$ & $\begin{array}{l}(\mathrm{m}) \\
(\mathrm{m}) \\
(-) \\
(\mathrm{MWD} / \mathrm{MTOx}) \\
(\mathrm{Pa})\end{array}$ & $\begin{array}{l}\text { fabricated inside clad radius } \\
\text { fabricated fuel radius } \\
\text { fabricated fuel porosity } \\
\text { fuel burnup induced } \\
\text { densification rate } \\
\text { initial fill gas pressure }\end{array}$ \\
\hline$t_{\mathrm{F}}$ & (h) & $\begin{array}{l}\text { time (length of time since } \\
\text { start-up) }\end{array}$ \\
\hline$q_{\mathrm{L}}$ & $(W / m)$ & $\begin{array}{l}\text { linear heat generation rate } \\
\text { on current time step }\end{array}$ \\
\hline $\begin{array}{l}p_{\mathrm{O}} \\
T_{\mathrm{CO}}\end{array}$ & $\begin{array}{l}(\mathrm{Pa}) \\
\left({ }^{\circ} \mathrm{C}\right)\end{array}$ & $\begin{array}{l}\text { outer pressure on clad } \\
\text { temperature of clad outer } \\
\text { radius }\end{array}$ \\
\hline $\begin{array}{l}R_{\mathrm{CO}, \mathrm{fab}} \\
\alpha_{\mathrm{q}}\end{array}$ & $\begin{array}{l}(\mathrm{m}) \\
(-)\end{array}$ & $\begin{array}{l}\text { fabricated clad outside radius } \\
\text { scale factor on entire linear } \\
\text { power history }\end{array}$ \\
\hline
\end{tabular}

a For Zircaloy clad, circumferential creep strain is renormalized to avoid an infinite primary creep rate at startup according to the transformation $\epsilon_{\theta, \mathrm{c}}^{*}=\epsilon_{\mathrm{eq}, \mathrm{th}}^{1 / m} \epsilon_{\theta, \mathrm{c}}$ where $\epsilon_{\theta, \mathrm{c}}$ is the clad circumferential creep strain, $\epsilon_{\mathrm{eq}, \mathrm{th}}$ is the equivalent thermal creep strain, and $m$ is the exponent in the time hardening rule $(m=0.3)$.

b Unrestrained dimensions include all effects except PCMI. In particular, negative unrestrained fuel/clad gap is allowed and signals the existence of contact pressure.

c Parameters below this line comprise the balance of input parameters used in SCODE.

$t_{\mathrm{g}}$ = fuel-clad gap,

$\lambda_{\mathrm{g}}=$ extrapolation length,

$\delta_{\mathbf{g}}=$ fuel-clad roughness,

$p_{\mathrm{c}}=$ fuel-clad contact pressure,

$a_{\mathrm{g}}=$ contact pressure coefficient.

The stainless steel fuel rod gap remains open throughout its life. Hence, an infinitesimal increase in as-fabricated fuel radius increases the gap conductance (by decreasing the gap size) which acts, together with an increase in the hot fuel radius, $R_{\mathrm{F}}$, to decrease $T_{\mathrm{F}}$ and hence $T_{0}$. Thus the sensitivity of $T_{0}$ with respect to $R_{\mathrm{F}, \text { fab }}$ in the case of an open gap is straightforward. 


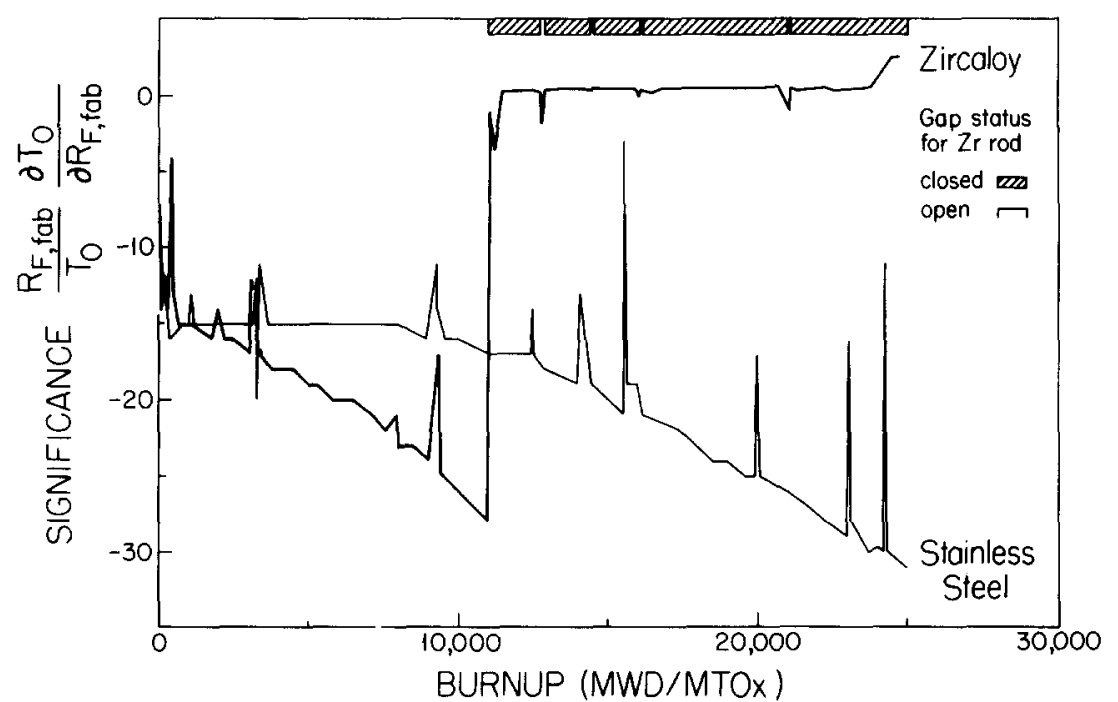

Fig. 2. Significance of fuel centerline temperature to fuel fabricated radius for type $B$ fuel and power history \#2.

In the case of a Zircaloy rod, the clad creepdown rate is much higher resulting in a rise in the significance at gap closure. This power history results in a condition where the fuel and clad are "lightly" touching ( $p_{\mathrm{c}}$ is small compared to the pressure due to the coolant) as evidenced by the flutter in gap status. In this case, an increase in $R_{\mathrm{F}, \mathrm{fab}}$ produces a decrease in the internal gas pressure, $p_{\mathrm{I}}$. This decrease in $p_{1}$ results from the smaller volume and hence fewer moles of $\mathrm{He}$ fill gas (for constant fill gas pressure) as $R_{\text {F.fab }}$ is increased. The lower gas pressure causes an increase in the value of the extrapolation length which causes a decrease in $h_{\mathrm{g}}$ and hence, an increase in $T_{0}$. Further, since the gap is closed, $t_{\mathrm{g}}$ is zero and does not contribute as in the open gap case. These dependencies are explained in the following expansion of the sensitivity. The arrows indicate that term 1 dominates in the open gap case where $t_{\mathrm{g}}^{\prime}>0$, but term 2 is dominant when $t_{\mathrm{g}}^{\prime}$ goes to zero.

$$
\begin{aligned}
& \frac{\partial T_{0}}{\partial R_{\mathrm{F}, \mathrm{fab}}}=\frac{\partial T_{0}}{\partial t_{\mathrm{g}}^{\prime}} \frac{\partial t_{\mathrm{g}}^{\prime}}{\partial R_{\mathrm{F}, \mathrm{fab}}}+\frac{\partial T_{0}}{\partial h_{\mathrm{g}}} \frac{\partial h_{\mathrm{g}}}{\partial \lambda_{\mathrm{g}}} \frac{\partial \lambda_{\mathrm{g}}}{\partial p_{\mathrm{I}}} \frac{\partial p_{\mathrm{I}}}{\partial R_{\mathrm{F}, \mathrm{fab}}} \\
& \begin{array}{l}
\text { Open gap: } \\
(-) \underbrace{(+)(-)}+(-)(-)(-)(-)
\end{array} \\
& \begin{array}{l}
\text { Closed gap: } \\
++\underbrace{(-)(-)(-)(-)}_{0}
\end{array} \\
& +\frac{\partial T_{0}}{\partial p_{\mathrm{c}}} \frac{\partial p_{\mathrm{c}}}{\partial R_{\mathrm{F}, \mathrm{fab}}}+\ldots \\
& +\quad \begin{array}{c}
0 \\
+\quad(-)(+) .
\end{array}
\end{aligned}
$$

This example services to illustrate the importance of internal gas pressure in a closed gap condition as well as the highly non-intuitive behavior of fuel centerline temperature with respect to fabricated fuel radius. From this analysis it can be concluded that the effect of an infinitesimal change in the as fabricated fuel radius on centerline temperature is appreciable and highly dependent on the gap status.

\subsection{Sensitivity of clad circumferential stress to fabricated clad inner radius, $\delta \sigma_{\theta} / \delta R_{\mathrm{Cl}, \text { fab }}$}

The mechanical behavior of the cladding is principally determined by the circumferential stress since this is typically the largest in magnitude of the principle stresses. The significance of circumferential clad stress with respect to as-fabricated inner clad radius is plotted in fig. 3 for Zircaloy and stainless steel clad fuel using power history 2, fuel type B. Note that in both cases when the gap is open, the significance is positive and constant. When the gap closes, as in the Zircaloy case, the significance becomes large and negative. The circumferential stress is defined using the thin shell approximation as

$\sigma_{\theta}=\frac{1}{2} \frac{R_{\mathrm{CO}}+R_{\mathrm{Cl}}}{R_{\mathrm{CO}}-R_{\mathrm{Cl}}}\left(p_{\mathrm{I}}-p_{\mathrm{O}}+p_{\mathrm{C}}\right)$,

where

$\sigma_{\theta}=$ clad circumferential stress,

$R_{\mathrm{CO}}=$ clad outer radius,

$R_{\mathrm{Cl}}=$ clad inner radius,

$p_{\mathrm{I}}=$ internal pressure,

$p_{O}=$ coolant pressure, 


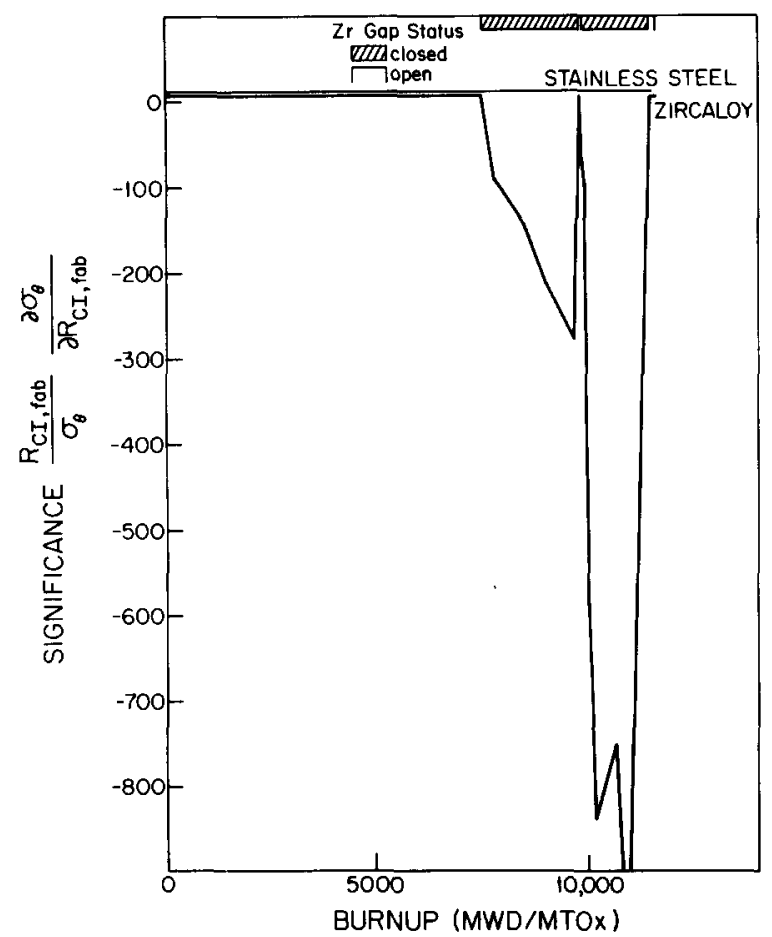

Fig. 3. Significance of clad circumferential stress to the asfabricated clad inner radius for type B fuel and power history \#2.

$p_{\mathrm{c}}=$ fuel-clad contact pressure.

Some helpful auxiliary definitions are

$p_{\mathrm{c}}=\frac{R_{\mathrm{F}}^{\prime}-R_{\mathrm{CI}}}{R_{\mathrm{F}}\left(w_{\mathrm{f}}+w_{\mathrm{c}}\right)}$,

and

$w_{\mathrm{c}}=\frac{R_{\mathrm{CI}, \mathrm{fab}}^{2}\left(1-\nu_{\mathrm{c}}\right)+R_{\mathrm{CO}, \mathrm{fab}}\left(1+\nu_{\mathrm{c}}\right)}{E_{\mathrm{c}}\left(R_{\mathrm{CO}, \mathrm{fab}}^{2}-R_{\mathrm{CI}, \mathrm{fab}}^{2}\right)}$,

where

$R_{\mathrm{F}}^{\prime}=$ unrestrained hot fuel radius,

$R_{\mathrm{F}}=$ restrained hot fuel radius,

$w_{\mathrm{f}}=$ fuel stiffness,

$w_{\mathrm{c}}=$ clad compliance,

$\nu_{\mathrm{c}}=$ poisson's ratio for the clad,

$E_{\mathrm{c}}=$ elastic modulus for the clad.

In the open gap case, $p_{\mathrm{c}}=0$ and $p_{0}>p_{\mathrm{I}}$ so the stress is negative and an infinitesimal increase in $\boldsymbol{R}_{\mathrm{Cl}, \mathrm{fab}}$ leads to a decrease in $R_{\mathrm{Cl}}$ because $\partial R_{\mathrm{Cl}} / \partial R_{\mathrm{Cl}, \text { fab }}<0$. But the change in $R_{\mathrm{CI}, \text { fab }}$ produces an even larger decrease in $R_{\text {co }}$ so the net result is an increase in the magnitude of the term $\left(R_{\mathrm{CO}}+R_{\mathrm{CI}}\right) /\left(R_{\mathrm{CO}}-R_{\mathrm{CI}}\right)$. This produces an even larger negative stress, giving rise to a negative sensitivity. Because of the negative sign of the stress, the significance is positive. Since $p_{0}$ is fixed and a change in $R_{\text {CI }}$ has only a secondary effect on $p_{1}$, the significance will remain nearly constant in the open gap case.

In the closed gap case the effect of contact pressure must be considered. An increase in $R_{\text {CI,fab }}$ would lead to an increase in the contact pressure. This happens because

$$
\begin{aligned}
\frac{\partial p_{\mathrm{c}}}{\partial R_{\mathrm{Cl}, \mathrm{fab}}} & =\frac{\partial p_{\mathrm{c}}}{\partial t_{\mathrm{g}}^{\prime}} \frac{\partial t_{\mathrm{g}}^{\prime}}{\partial R_{\mathrm{Cl}}} \frac{\partial R_{\mathrm{Cl}}}{\partial R_{\mathrm{CI}, \mathrm{fab}}} \\
(+) \quad & =(-)(+) \quad(-)
\end{aligned}
$$

The magnitude of this term is such that it dominates the expression for $\sigma_{\theta}$ resulting in a positive sensitivity and a negative significance (because of the sign on $a_{\theta}$ ). The following sensitivity expansion helps to explain the contribution of each of the variables in the relation for $\sigma_{\theta}$.

$\frac{\partial \sigma_{\theta}}{\partial R_{\mathrm{CI}, \mathrm{fab}}}=\frac{\partial \sigma_{\theta}}{\partial R_{\mathrm{CI}}} \frac{\partial R_{\mathrm{CI}}}{\partial R_{\mathrm{CI}, \mathrm{fab}}}+\frac{\partial \sigma_{\theta}}{\partial R_{\mathrm{CO}}} \frac{\partial R_{\mathrm{CO}}}{\partial R_{\mathrm{Cl}, \mathrm{fab}}}$

Open gap:

$$
\begin{aligned}
& (-) \quad(-)(-)+(+)(-) \\
& \text { Closed gap: } \\
& (+) \quad(-)(-)+(+)(-) \\
& +\frac{\partial \sigma_{\theta}}{\partial p_{\mathrm{I}}} \frac{\partial p_{\mathrm{I}}}{\partial R_{\mathrm{Cl}, \mathrm{fab}}}+\frac{\partial \sigma_{\theta}}{\partial p_{\mathrm{C}}} \quad \frac{\partial p_{\mathrm{C}}}{\partial R_{\mathrm{Cl}, \mathrm{fab}}}, \\
& +(+)(+)+0 \text {, } \\
& +(+)(+)+\underbrace{(+)(+) .}
\end{aligned}
$$

Note that term \#2 is dominant in the open gap case but term \#4 dominates when the fuel and clad touch.

\subsection{Sensitivity of gap size to fabricated clad inner radius} $\delta t_{\mathrm{g}}^{\prime} / \delta R_{\mathrm{CI}, \mathrm{fab}}$

The significance of gap thickness with respect to fabricated inner clad radius is plotted in fig. 4 for Zircaloy cladding using type A fuel and power history 1. Note that the significance increases sharply in magnitude with burnup (decreasing gap size). It is clear that the as-fabricated clad radius should have a large effect on the subsequent gap size. The large magnitudes shown in fig. 4 are due to the fact that as the gap gets smaller, a small fractional change in clad radius will produce a much larger fractional change in the gap size. Such a sensitivity is useful in controlling gap closure via linear power.

If the sensitivity of gap size to linear power $\left(\delta t_{\mathrm{g}}^{\prime} / \delta q_{\mathrm{L}}\right)$ were known, then one could estimate at any point in the 


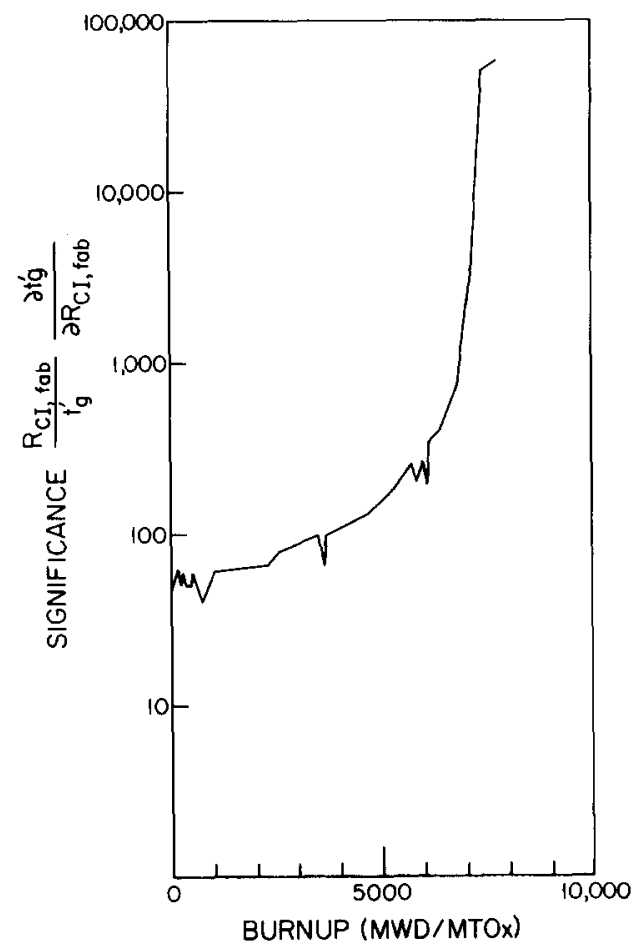

Fig. 4. Significance of gap thickness to as-fabricated clad inner radius for Zircaloy clad using fuel type $A$ and power history \#1.

power history, the power step needed to close the gap on the next time step. The sensitivity of interest [7] is

$$
\frac{\delta R_{\mathrm{F}}^{\prime}}{\delta q_{\mathrm{L}}}=\frac{F_{\text {dep }}}{\pi k_{\mathrm{f}}\left(T_{\mathrm{A}}\right)}\left[\frac{b}{12}+\frac{c T_{\mathrm{F}}}{6}+\frac{c F_{\text {dep }} q_{\mathrm{L}}}{40 k_{\mathrm{f}}\left(T_{\mathrm{A}}\right)}\right],
$$

where $F_{\text {dep }}=$ flux depression factor, $b, c=$ constants from the expression for fuel thermal expansion, $T=a+$ $b T+c T^{2}$, and all other terms are as previously defined. Knowing both radial and axial power peaking factors, the effect of power maneuvers on the gap status of rods throughout the core can be ascertained. Since, as we have seen, the sensitivities of many output variables change radically in sign and/or magnitude upon gap closure, such an estimation becomes very useful in fuel performance modeling.

\section{Zircaloy vs. stainless steel cladding}

The capability of modeling two cladding types allows the performance of Zircaloy and stainless steel fuel cladding to be compared at different points in the power history. Referring to runs using history 1 for both cladding types, $\mathrm{Zr}$ and SS clad rods show nearly identical sensitivities to the input parameters at the beginning of life. This is not unexpected since the sensitivities depend both on rod history and current rod conditions (via the $[A]$ matrix). Since rod conditions are nearly identical at start-up, similar rods will have similar dependencies for a common initial power step. However, as burnup proceeds, a significant divergence occurs in three of the sensitivities. After a burnup of approximately $5000 \mathrm{MWD} / \mathrm{MTOx}, \partial G_{\mathrm{Rel}} / \partial R_{\mathrm{Cl}, \mathrm{fab}}, \partial T_{0} / \partial p_{\mathrm{I} \text {,fab }}$ and $\partial \sigma_{\theta} / \partial R_{\mathrm{F}, \text { fab }}$ all increase in the $\mathrm{Zr}$ case relative to the SS case. The reason for this is in the higher creep rate of Zircaloy. For a given set of input and operating conditions, the creep rate of $\mathrm{Zr}$ is greater than the creep rate of SS. In the open gap case then, the gap will shrink faster for $\mathrm{Zr}$ than for SS under the identical power history. The variables affected most by this changing gap size will be fission gas release, fuel temperatures and circumferential stress. Likewise, the parameters $R_{\mathrm{CI}, \mathrm{fab}}$ and $R_{\mathrm{F}, \mathrm{fab}}$ exert the strongest effects on the output variables and $p_{\text {I,fab }}$ the next strongest, table 5 . Therefore,due to its inherently greater creep rate $\mathrm{Zr}$ clad rods experience larger sensitivities than SS clad rods under similar operating conditions. These conclusions are valid, however, only at or about a burnup of 5000 MWD/MTOx. Since the fuel rod is a dynamic system in which gap status may be constantly changing, the principal sensitivities will also change. The sensitivities of each fuel type must be monitored throughout the entire power history to obtain a complete description of their comparative performance.

Table 5

Ranking of input parameters according to their effect on key output variables

\begin{tabular}{lll}
\hline Rank & Parameter & Range of $\partial V_{i} / \partial P_{k}{ }^{\mathrm{a}}$ \\
\hline & $\left\{\begin{array}{l}R_{\mathrm{Cl}, \mathrm{fab}} \\
R_{\mathrm{CO}, \mathrm{fab}} \\
R_{\mathrm{F}, \mathrm{fab}}\end{array}\right\}$ & $1-10^{2}$ \\
2 & $\left\{\begin{array}{l}P_{\mathrm{fab}} \\
\alpha_{\mathrm{q}} \\
q_{\mathrm{L}} \\
T_{\mathrm{CO}} \\
p_{\mathrm{I}, \mathrm{fab}} \\
P_{\mathrm{O}}\end{array}\right)$ \\
3 & $\left\{\begin{array}{l}t_{\mathrm{F}} \\
\text { bu den }\end{array}\right\}$ & $10^{-2}-1$ \\
\hline
\end{tabular}

a refers to the 5 key output variables given in Table 4 . 


\section{Ranking the input parameters}

The importance of the input parameters as they affect the 5 selected output variables is given in table 5 . For both Zircaloy and stainless steel clad fuel, the most important input parameters are $R_{\mathrm{CI}, \mathrm{fab}}, R_{\mathrm{CO}, \mathrm{fab}}$, and $R_{\text {F,fab }}$. The significances of all five output variables to these parameters are greater than unity throughout the power history and approach a value of 100 . This means that dimensional control of fuel tubes and fuel pellets is of greatest importance and errors in the tolerance will be reflected most acutely in the stress and strain rate in the clad, along with the fuel temperature, gas release and gap size. Of least importance is the time and the fuel burnup densification rate, $\mathrm{bu}_{\mathrm{den}}$. Intermediate to these categories are the fabricated fuel porosity, the linear heat generation rate at the current time step, the scale factor on the entire linear power history, the clad outer temperature, the initial fill gas pressure, and the coolant pressure. These findings hold particular importance for the fuel designer and manufacturer.

An example is the fabrication of a fuel pellet. A reasonable value for the tolerance as a fraction of the fuel radius is $0.73 \%$. The significance $\left(\delta T_{0} / T_{0}\right) /$ $\left(\delta R_{\mathrm{F}, \mathrm{fab}} / R_{\mathrm{F}, \mathrm{fab}}\right)$ for both the stainless steel and Zircaloy clad rods is of the order -15 early in life (fig. 2). Thus $\delta T_{0} / T_{0}$ is 0.11 giving a $\delta T_{0}$ of $110^{\circ} \mathrm{C}$ for a fuel centerline temperature of $1000^{\circ} \mathrm{C}$. Later in life, the value of this significance can reach levels almost three times the early-in-life level causing large errors in the computed value of $T_{0}$.

It should be noted that the sensitivity $\partial X / \partial p$ represents a change in $X$ given an infinitesimal change in $p$. Hence, in the above example such macroscopic changes in the input parameter may not result in the computed change in the variable. Nevertheless, the magnitude of this derivative illustrates that control of fuel and clad dimensions is extremely important. Although it may be expected that rod component dimensions are important, it is somewhat surprising that the fuel characteristics do not appear in this upper category. Table 5 indicates that fuel porosity and densification rate are of secondary importance.

\section{Conclusions}

Adjoint sensitivity analysis is extended to operate on the entire power history of a fuel rod. FCODE-SCODE runs are made on two types of fuel and two types of fuel cladding, Zircaloy and stainless steel, and over two power histories. Several examples are presented illustrat- ing the non-intuitive behavior of sensitivities throughout the power history. The input parameters are classified according to the magnitude of their effect on five input variables; clad circumferential creep strain, clad circumferential stress, fission gas release, fuel centerline temperature and gap thickness. The principal results of this study are as follows:

- The most important input parameters are the fuel and cladding dimensions. Errors in dimensional control of these parameters that are of the magnitude of the tolerance limit $(\sim 1 \%)$ can produce errors in the centerline temperature in excess of $11 \%$, assuming that the derivative $\partial X / \partial p$ is valid for a region $\pm 0.01 p$ about $p$.

- Fuel characteristics such as total porosity and burnup densification rate are secondary to rod dimensions in their effect on the key output variables.

- The sensitivities of several key variables are greater for Zircaloy clad rods than stainless steel clad rods due to the inherently higher creep rate of Zircaloy, making that system more responsive to changes in input parameters.

- The importance of fuel-clad gap status is highlighted by sensitivity analysis. Gap closure, more often than not, produces large swings in the magnitudes of the sensitivities as well as changes in their signs.

- Because of the large number (33) of interrelated variables and the process by which sensitivities are determined (simultaneous solution of 33 equations), they are often highly non-intuitive. However, the capability of uncovering dependencies that cannot be determined by a sequential thought process is precisely where the value and power of this too lies.

- Sensitivity analysis is performed on all of the equations in the fuel behavior model simultaneously and in one code run. The same amount of information obtained by conventional methods (altering one input parameter each code run) would require a number of codes runs equal to the number of input parameters. Even so, the output is dependent on the size of the adjustment in the input parameter (secant method) whereas adjoint sensitivity analysis always yields a derivative (tangent method).

The data presented here represent only a small fraction of that produced in every FCODE-SCODE run. The examples discussed are intended only as a demonstration of the unique value and utility of adjoint sensitivity analysis. In this sense, the analysis is entirely incomplete. A complete categorization of trends and dependencies is impossible due to the nearly infinite combination of input parameters and power histories that may be applied to a fuel rod system. However, the 
major trends of sensitivities with power history and cladding type have been established for five key output variables which are of universal importance in the performance of any power reactor fuel rod. The main value of this technique lies in its application to specific cases where a greater understanding between fuel variables and input conditions is sought.

\section{Acknowledgement}

The authors gratefully acknowledge Ron Christensen at Entropy LTD for his many insightful discussions, and Northeast Utilities Service Co. for financial support of this project.

\section{References}

[1] D.R. Harris and M. Becker, Nonlinear perturbation technique for the nuclear fuel cycle, Trans. Amer. Nucl. Soc. 23 (1976) 534.
[2] D.R. Harris, Sensitivity of nuclear fuel cycle costs to uncertainties in nuclear data and methods, Doctoral Thesis, Rensselaer Polytechnic Institute (1976).

[3] R.A. Christensen, R.F. Eilbert, R.A. Rohrer and G.S. Was, Adjoint sensitivity analysis in nuclear reactor fuel behavior modeling, Nucl. Engrg. Des. 66 (1981) 125.

[4] R.A. Christensen, SPEAR fuel reliability code system: general description; Electric Power Research Institute, Interim Report EPRI NP-1378 (March 1980).

[5] G.S. Was, Fuel performance evaluation package for Connecticut Yankee, University of Michigan, Interim Progress Report, UMNMP-1 (Sept. 1982).

[6] R.A. Christensen, Sensitivities benchmarking, Entropy LTD., Interim Report (March 1979).

[7] R.A. Christensen, Fuel rod mechanical performance modeling; Task 3: fuel rod modeling decision analysis, Electric Power Research Institute, Eighth Quarterly Progress Report, RFMPM31-1 (May 1979) pp. 5-18 to 5-21. 